\title{
Factores desencadenantes de violencia dirigida a personal de salud de un hospital de segundo nivel de atención
}

\author{
Puente-Rojas. Ana Ruthi; Morales-Álvarez, Clara Teresita2*; Moreno-González, María Mercedes³; \\ Cerrito-Rodríguez, Gabriela4; Pérez-Lerma, Roxana de la Trinidad ${ }^{5}$
}

\section{RESUMEN}

Introducción: Los profesionales de la salud enfrentan frecuentemente situaciones de violencia durante el desempeño de sus funciones con riesgo de desarrollar consecuencias físicas, psicológicas y laborales. Objetivo: Determinar los factores que desencadenan violencia hacia el personal de salud. Metodología: Estudio descriptivo transversal, con una muestra de 66 personas en un hospital de segundo nivel de atención en México, se realizó un muestreo no probabilístico, se utilizó el instrumento "Agresiones hacia profesionales en el ámbito de la salud". En cada etapa del estudio se atendieron los principios éticos de acuerdo con el Reglamento de la Ley General de Salud en Materia de Investigación y declaración de Helsinki. Para el análisis de los datos se empleó el programa SPSS versión 20 y se aplicó estadística descriptiva. Resultados: Los factores desencadenantes de violencia hacia el personal, fueron la carencia de recursos materiales que se encontró presente en el $40 \%$ de los casos, el comunicado del fallecimiento del paciente en el 30\%, la demora de la atención en un 20\%. El principal agresor fue el paciente. El 96\% del personal no abandonó su labor después de sufrir el acto de violencia. Conclusiones: Los principales desencadenantes de violencia corresponden a aspectos administrativos de material y recursos, esta situación debe atraer la atención de las autoridades sanitarias ya que es de su competencia la gestión del recurso material y humano.

\section{Palabras clave: Violencia, hospitales, personal de salud (DeCS; BIREME)}

\begin{abstract}
1. Licenciada en Enfermería y Obstetricia. Universidad de Guanajuato, Campus Celaya - Salvatierra, División de Ciencias de la Salud e Ingenierías. Celaya Guanajuato México. Correo electrónico: anapuentepr@hotmail.com

2.Maestra en Ciencias de Enfermería. Universidad de Guanajuato, Campus Celaya-Salvatierra, División de Ciencias de la Salud e Ingenierías. Celaya Guanajuato México. Correo electrónico: tmorales@ugto.mx ORCID ID: orcid.org/0000-0002-5943-9048

3.Maestra en Ciencias de Enfermería. Universidad de Guanajuato, Campus Celaya - Salvatierra, División de Ciencias de la Salud e Ingenierías. Celaya Guanajuato México. Correo electrónico: ma.moreno@ugto.mx. ORCID ID: orcid.org/0000-0002-0299-2299

4.Licenciada en Enfermería y Obstetricia. Universidad de Guanajuato, Campus Celaya - Salvatierra, División de Ciencias de la Salud e Ingenierías. Celaya Guanajuato México. Correo electrónico: gabizerrito@gmail.com

5. Licenciada en Enfermería y Obstetricia. Universidad de Guanajuato, Campus Celaya - Salvatierra, División de Ciencias de la Salud e Ingenierías. Celaya Guanajuato México. Correo electrónico: roxanaperezlerma@gmal.com
\end{abstract}

Recibido: $16 / 11 / 2018$

Aceptado: $11 / 12 / 2018$

${ }^{*}$ Autor para correspondencia

\section{Cómo citar este artículo}

Puente-Rojas AR, Morales-Álvarez CT, Moreno-González MM, Cerrito-Rodríguez G, Pérez-Lerma RT. Factores desencadenantes de violencia dirigida a personal de salud de un hospital de segundo nivel de atención. SANUS. 2018;(8): 26-36. [Acceso_____]; Disponible en: 


\title{
Fatores desencadeantes de violência dirigida ao pessoal de saú- de de um hospital de cuidados de segundo nível
}

\begin{abstract}
RESUMO
Introdução: Os profissionais de saúde freqüentemente experimentam situação de violência durante o desempenho de suas funções com o risco de desenvolver conseqüências físicas, psicológicas e trabalhistas. Objetivo: Determinar los fatores que desencadeiam violência contra o pessoal de saúde. Metodologia: Estudo descritivo transversal, com uma amostra de 66 pessoas em um hospital de segundo nível de atendimento no México. Uma amostragem não probabilística foi realizada, o instrumento foi usado "Agressões contra profissionais no campo da saúde". Em cada etapa do estudo eles foram atendidos os princípios éticos de acordo com o Regulamento da Lei Geral de Saúde sobre Pesquisa e Declaração de Helsinque. Para a análise dos dados foi utilizada a versão 20 do programa SPSS e estatística descritiva foi aplicada. Resultados: Os fatores que desencadeiam a violência para o pessoal foram a falta de recursos materiais que esteve presente em $40 \%$ dos casos, a declaração da morte do paciente em 30\% atraso de atendimento em 20\%. O principal agressor foi o paciente. 96\% do pessoal não desistiu do trabalho depois de sofrer o ato de violência. Conclusões: Os principais desencadeantes da violência correspondem a aspectos administrativos, materiais e recursos, esta situação deve atrair a atenção das autoridades de saúde já que é de sua competência a gestão dos recursos materiais e humanos.
\end{abstract}

Palavras chave: Violência, hospitais, pessoal de saúde (DeCS; BIREME).

\section{INTRODUCCIÓN}

Los profesionales sanitarios enfrentan continuamente situaciones de violencia verbal y física durante el ejercicio de sus labores, lo que tiene consecuencias en el ámbito laboral y de salud (1).

La Organización Mundial de la Salud (OMS), el Consejo Internacional de Enfermeras (CIE), la Organización Internacional del Trabajo (OIT) y la Organización Internacional de Servicios Públicos (ISP) definen violencia laboral como: Incidentes en los que el personal sufre abusos, amenazas o ataques en circunstancias relacionadas con su trabajo (2).

Diversas investigaciones arrojan cifras significativas acerca de actos de violencia dirigida a personal de salud ${ }^{(3-5)}$. En un estudio realizado en España se muestra que 75.9\% de los participantes refieren haber sido víctimas de agresiones a lo largo de su ejercicio profesional, sin encontrar diferencias significativas entre sexo y tipo de agresión ${ }^{(6)}$. Mientras que en Argentina se encontró que el $66.7 \%$ de los participantes externaron algún tipo de agresión en sus centros laborales en el último año. Los más agredidos fueron los enfermeros en $71.6 \%$ y los médicos con $71.3 \%$, el resto de las ocupaciones presentaron tasas inferiores. Los factores que causaron agresiones fueron la comunicación de fallecimientos, atención a pacientes intoxicados o con estado mental alterado ${ }^{(5)}$. Así mismo, reportaron que la violencia física se asocia significativamente con un mayor riesgo de presentar complicaciones en comparación con las agresiones verbales. El contexto de la mayoría de los episodios de agresión fue en las áreas de emergencias ${ }^{(5)}$.

La violencia en instituciones de salud ya sea física o psicológica, llega a tener efectos negativos sobre los trabajadores que son víctimas de ella y en consecuencia reduce la calidad en el desempeño de sus tareas ya que causa desmotivación al ejercer el cuidado, un ejemplo de ello es que el personal de enfermería que desempeñó su trabajo en un ambiente estresante presentó una menor satisfacción con el mismo ${ }^{(7,8)}$.

Las múltiples formas de violencia estructural determinan el ejercicio de violencia directa, física y psicológica y su impacto negativo en la salud de los trabajadores ${ }^{(9)}$. Por lo tanto, los estragos de violencia, se puede presentar a la par que la frecuencia de su práctica. En España el 68.3\% de los enfermeros declararon haber sufrido una agresión, siendo la violencia verbal la de mayor frecuencia en 37,2\%, seguido por 18.6\% en la combinación de agresión verbal y física, 6.9\% en la psicológica y física con 4.8\% (1).

Con respecto al personal médico, en Pakistán 1 de cada 6 han sufrido ataque físico y 3 de cada 5 abusos verbales, aquellos que informaron haber sufrido ataque físico fueron 6.7 veces más propensos a reportar estrés postraumático ${ }^{(10)}$. 
Como se observa en los estudios anteriores, la agresión entre las personas ha transgredido todas las barreras, introduciéndose a las instituciones de salud, en dónde debe procurarse un ambiente neutro y confortable ya que son espacios de sanación.

No obstante, todos los días acontecen posibles riesgos de violencia; la aglomeración en las salas hospitalarias, escasez de personal, la presencia de urgencias sentidas por parte del paciente, pacientes que reciben del médico una mala noticia, diagnóstico o pronóstico no esperado, así como la negación de una baja laboral temporal (1).

Son múltiples las causas de descontento entre el servidor sanitario y el paciente, en consecuencia, originan violencia, la cual en el sentido social es considerada como un problema de salud y un importante factor de riesgo psicosocial, y dada la magnitud del daño, puede dar lugar a la presencia de invalidez e incluso la muerte de la persona violentada (11).

Por lo anterior, se requiere realizar una profunda reflexión de las condiciones de seguridad laboral del personal de salud, sobre todo aquel que se encuentra en mayor contacto con pacientes o que brinda atención en los momentos más críticos en la salud de los pacientes, así como los mecanismos de afrontamiento a dichos eventos.

Ante esta situación, la calidad de la atención se ve afectada, no obstante, el Sistema Nacional de Salud en México, ha dado prioridad a la seguridad del paciente con la práctica de las acciones esenciales para la seguridad del mismo colocando a la calidad de prestación de servicios de salud en la agenda permanente del gobierno mexicano, estrategias que son relevantes y pertinentes para un cuidado humanizado y de calidad, asegurando la pronta reintegración de los pacientes a la sociedad. Lo anterior muestra el área de oportunidad para cuestionarse: ¿Qué sucede con la seguridad de los trabajadores sanitarios?, ¿Se respetan sus derechos?

Las interrogantes anteriores llevaron a conducir el objetivo de este trabajo para determinar los factores que desencadenan la violencia hacia el personal sanitario.

\section{METODOLOGÍA}

Estudio de tipo transversal descriptivo, retrospectivo. Dirigido a personal de salud de un nosocomio público de una ciudad del centro del país. Se calculó una muestra de 66 participantes a partir de la fórmula de población finita, con un I.C. de 95\%, proporción esperada y precisión del 5\%, el muestreo fue no probabilístico.

Se abordaron a varios profesionales de la salud, sin embargo solo se incluyeron a trabajadores de salud que refirieron haber sido violentados en los últimos seis meses, se les explicó el objetivo y se atendieron sus inquietudes, posteriormente firmaron el consentimiento informado, y se inició la colecta de datos a través del cuestionario autoadministrado "Agresiones hacia profesionales en el ámbito de la salud"(5), conformado por 16 ítems el cual obtuvo una confiabilidad de 0.89 por Kappa.

En cada etapa del procedimiento se atendieron las consideraciones éticas: de acuerdo a lo establecido por el Reglamento de la Ley General de Salud en Materia de Investigación para la Salud ${ }^{(12)}$, se consideró el Artículo 13 en relación al respeto a la dignidad, protección de los derechos y bienestar de los participantes. De acuerdo al Artículo 14, la investigación fue sometida a revisión y aprobada por el Comité Científico y de Investigación del Hospital General de Celaya, con el número de registro 39/2017. Una vez identificados los trabajadores de la salud que refirieron haber sido violentados en los últimos 6 meses se atendió el artículo 22, para ello se elaboró una carta de consentimiento informado y de acuerdo al artículo 16 se protegió su privacidad, conservando su anonimato.

Los datos fueron analizados con el programa SPSS versión 20, se utilizó estadística descriptiva para las variables categóricas, se calculó frecuencias y porcentajes para las variables numéricas, medidas de tendencia central y dispersión.

\section{RESULTADOS}

Se entrevistaron 66 profesionales de salud, con edad media de 33 años $(D E=7.2)$, predominio de sexo femenino con 63.6\%. La profesión más violentada fue la de Licenciados en Enfermería, seguido de la de Licenciados en Medicina (Figura 1). Del total de los participantes, 36\% de ellos fueron violentados por lo menos en 2 ocasiones en los últimos 6 meses. El área dónde se presentó con mayor frecuencia la agresión fue en hospitalización (56\%).

El tipo de agresión más frecuente es la verbal por parte del paciente (50\%) y de los familiares (33\%), así mismo la violencia física por parte de los pacientes se presentó en $17 \%$.

Los principales factores desencadenantes de violencia identificados fue la carencia de recursos, el comunicado del fallecimiento del paciente, la demora de la atención y debido al informe médico (Figura 2).

El 50\% del personal de salud refiere haber sufrido ansiedad después de los hechos, 33\% presentó síndrome de Burnout y el 17\% lesiones físicas. Un aspecto que vale la pena reconocer es que el 96\% del personal que reportó haber sufrido de algún tipo de agresión no abandonó ni suspendió su tarea asistencial. 33\% del personal de salud al ser agredido trató de usar el diálogo para controlar la situación, y 27\% avisó a otros trabajadores.

\section{DISCUSIÓN}

Diversos autores coinciden en que la violencia hacia el personal tiene una alta prevalencia en las instituciones de salud ${ }^{(13,14)}$, en este estudio resultó que el personal de 
Figura 1. Personal de salud que sufrió violencia.

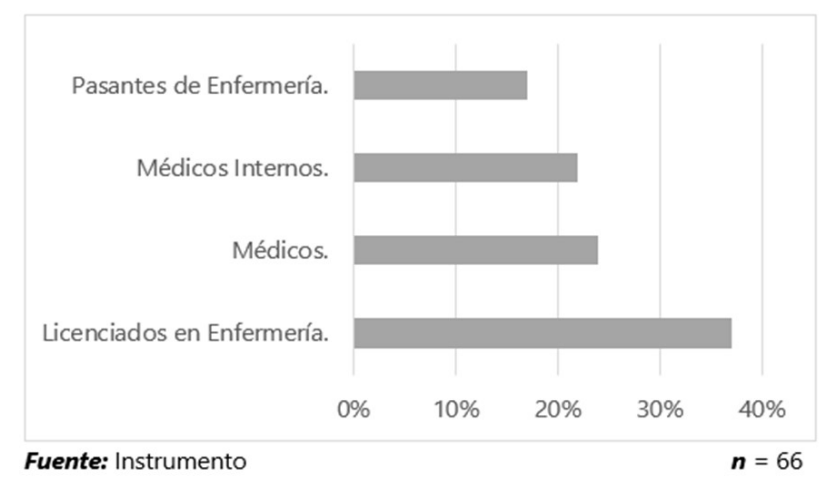

enfermería es más agredido que el resto de los profesionales, lo que concuerda con el estudio de Bordignon y Monteiro en donde el $81.8 \%$ del personal de enfermería manifestó haber sido agredido y el $42 \%$ manifestó haber sufrido violencia verbal al menos una vez en el último año por parte del paciente ${ }^{(15)}$, de igual forma en un hospital italiano el 11.5\% tenía experiencia de violencia física y el $40.2 \%$ había sido víctima de violencia verbal en los últimos 12 meses ${ }^{(16)}$, en contraste el $36 \%$ los sujetos de este estudio fueron agredidos dos veces en un período de seis meses, por lo que puede inferirse que en México la violencia al personal de salud es más frecuente o bien que se encuentra en aumento.

En los resultados encontrados en el presente estudio, se puede apreciar que la violencia dirigida a los trabajadores de la salud en el contexto mexicano sigue un patrón similar al de otros países. En diversos hospitales de la ciudad de Córdoba, Argentina el 62.9 \% reconocen la presencia de situaciones de violencia en su lugar de trabajo de las cuales los principales agresores fueron los familiares quienes gritaron y descalificaron el desempeño del personal médico ${ }^{(17,18)}$ situación contrastante con este estudio, dónde el principal agresor fue el paciente, con respecto al tipo de agresión, la verbal fue similar a lo reportado en Cuba en donde el $63.9 \%$ de los incidentes se describieron como violencia verbal y sólo el 11.9\% como violencia física ${ }^{(16)}$.

El estudio de Irinyi, et al. ${ }^{(13)}$ concuerdan con los resultados del presente estudio, en donde se hace énfasis en que el personal que más sufre agresión en el área hospitalaria es el personal de enfermería, y de manera general, los departamentos de emergencia son más vulnerables a la violencia ${ }^{(14)}$. En contraste en este estudio los enfermeros fueron agredidos con mayor frecuencia en las áreas de hospitalización.

Posterior a la agresión el personal reportó sentimientos de inseguridad relacionados con su rol profesional (16), no obstante, el personal de salud no se atreve a abandonar al usuario, sino que continua la atención. Tampoco se atreve a denunciarlo, posiblemente como consecuencia de que el personal violentado no recibió asesoría para denunciar la violencia ${ }^{(19)}$.
Figura 2. Factores desencadenantes de violencia

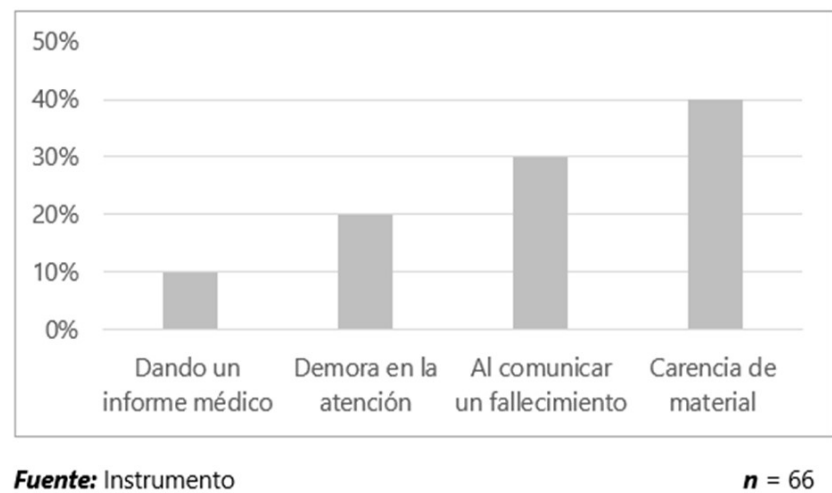

Así mismo, un alto porcentaje de trabajadores no reaccionaron frente a los actos violentos, pocos reportaron el incidente y nadie entabló demandas legales en ninguno de los tipos de violencia ${ }^{(20)}$.

La violencia puede ser originada por largas horas de espera, incertidumbre en el tratamiento y pronóstico ${ }^{(13)}$, en este estudio el factor de violencia hacia el personal que se presentó con más frecuencia fue la falta de material.

Sin embargo, el abasto de materiales no es de responsabilidad directa del personal operativo sino del personal directivo y administrativo, ante esta situación el personal debe ser capacitado para saber qué hacer en caso de violencia, así como generar agencias o módulos de quejas para emitir sanciones al usuario violento ya que uno de los derechos de los y las enfermeras de México es "Desempeñar sus intervenciones en un entorno que garantice la seguridad e integridad personal y profesional" (21).

Por otra parte, el cuidado de enfermería en ocasiones se ve afectado en la atención básica, por falta de recursos materiales en un 56\% (22), indiscutiblemente el recurso material es parte de la calidad del cuidado prestado por los enfermeros provocando sentimientos de ambivalencia entre los profesionales de la salud, es decir, la vocación de servicio se ve afectada ante las agresiones por parte de los sujetos de cuidado(23); las enfermeras enfrentan un reto al brindar atención dentro del entorno de la nueva gestión pública. Esto se puede ver en las tensiones que dibujan una línea entre el cuidado como una disciplina profesional y el cuidado como un objetivo financiero, asociadas al choque de valores que surge de la forma en que el cuidado de enfermería se conceptualiza en cada uno de los centros de trabajo ${ }^{(23)}$.

Los líderes y colegios de enfermería deben poner manos a la obra para someter a la legislación mexicana la seguridad laboral del personal de enfermería.

\section{CONCLUSIONES}

La violencia dirigida al personal de salud por parte del paciente es un acto frecuente que por distintas circunstancias 
es un tema al que no se le ha dado prioridad.

El manejo del usuario debe ser de calidad, considerar los factores desencadenantes de violencia, actuar con cautela y tomar las medidas apropiadas para el manejo de la situación, evitará verse implicado en cuestiones legales, así como sufrir lesiones o secuelas físicas y psicológicas.

Esto ayudará a que enfermeras, médicos, nutriólogos, entre otros profesionales gocen de una adecuada salud mental que no merme en el ejercicio de su profesión e indirectamente en el trato que el paciente recibe por parte de ellos.

La alta frecuencia de violencia ejercida hacia el personal de salud en el contexto mexicano debe atraer la atención de las autoridades sanitarias con el objetivo de emitir las acciones preventivas correspondientes.

\section{CONFLICTO DE INTERESES}

Los autores declaran no tener conflicto de interés.

\section{FINANCIAMIENTO}

Este trabajo fue autofinanciado por los autores.

\section{REFERENCIAS BIBLIOGRÁFICAS}

1. Martínez S, García A, Felipez I. Castro D. Violencia sufrida y percibida por el personal de enfermería del Área Sanitaria Integrada de A Coruña. Rev. Enfermería Global. 2015; 14(3):319-29. [consultado abril de 2018]. Disponible en: http://scielo.isciii.es/pdf/eg/v14n39/administracion2.pdf

2. Organización internacional del trabajo. Programa conjunto: nueva iniciativa contra la violencia laboral en el sector de la salud. Octubre 2015. [consultado septiembre de 2017]. Disponible en: http://www.lo.org/global/about-theilo/newsroom/news/WCMS_071411/lang--es/index.htm

3. Mayorca I, Lucena S, Cortés M, Lucerna M. Violencia contra trabajadores sanitarios en un hospital de Andalucía, ¿Por qué hay agresiones no registradas? Rev. Med. Segur. Trab 2013;59(231):235-58.

4. Galián I. Violencia de los usuarios hacia el personal de enfermería. Evaluación del riesgo laboral y análisis de las consecuencias psicológicas [Tesis doctoral] Universidad de Murcia, Facultad de Enfermería 2015. [consultado agosto de 2018]. Disponible en: http://www.tesisenred.net/bitstream/ handle/10803/300898/TIGM.pdf?sequence =1

5. Travetto C, Daciuk N, Fernández S, Ortiz P, Mastandueno $\mathrm{R}$, Prats $\mathrm{M}$, et al. Agresiones hacia profesionales en el ámbito de la salud. Rev Panam Salud Pública. 2015; 38(4):307-15.
6. Madrid PJ, Salas MJ, Madrid M. Situaciones de las agresiones a Enfermería en el área de la salud Puertollano. Rev. Enfermería del trabajo 2011;1(1):11-7.

7. Itzhaki M, Bluvstein I, Peles A, Kostistky H, Bar D, Filshtinsky $V$, and Theilla M. Mental Health Nurse's Exposure to workplace violence leads to job stress, which leads to reduced professional quality of life. Rev. Front Psychiatry 2018;9(59); 1-6.

8. Muñoz G, Zaragoza LI, Ruiz J, Jímenez J. Exposición a violencia de los usuarios y satisfacción laboral en el personal de enfermería de los hospitales públicos de la región de Murcia. An.Sist. Sant. Navar 2018;41(2):181-89.

9. Luna J, Urrego Z, Gutiérrez M, Martínez A. Violencia en el trabajo del sector público de la salud: una visión desde las personas trabajadoras. Bogotá, Colombia. 2011-2012 Rev Fac. Med. 2015; 63(3): 407-17.

10. Waleed Z, Uzma R, Siddiqui S, Jamali S, Razzak J. Workplace Violence and Self-reported Psychological Health: Coping with Post-traumatic Stress, Mental Distress, and Burnout among Physicians Working in the Emergency Departments Compared to Other Specialties in Pakistan. Rev. The Journal of Emergency Medicine 2016;50(1):167-77.

11. Araujo R y Diaz G. Un enfoque teórico-metodológico para el estudio de la violencia. Rev Cubana Salud Pública. 2000;24(2):85-90.

12. Diario Oficial de la Federación. Reglamento de la Ley General de Salud en Materia de Investigación para la Salud. México; 2014. [consultado agosto de 2018]. Disponible en: https://www.gob.mx/conamer/prensa/reglamento-de-laley-general-de-salud-en-materia-de-investigacion-para-lasalud

13. Irinyi $T$, Németh A, Lampek K. Violence Against health care providers and its correlations with sociodemographic and workplace-related factors. Int I Community Med Public Health. 2017;158(6):229-37.

14. Tadros A, Kifer C. Violence in the emergency Department: A Global Problem. Rev. Psychiatr. Clin. N. Am. 2017; 40 (3):575-84.

15.Bordignon M. Monteiro M. Violence in the work in Nursing consequences overview. Rev. Bras. Enferm. 2016; 69 (5): 93942.

16. Terzoni S, Gerrera P, Cornelli R, Ricci C, Oggioni C, Destrebecq A. Violence and unsafety in major Italian hospital: experience and perceptions of health care worker. Rev. Med. Lav. 2015; 106(6):403-11. 
17. Farías A, Sánchez J, Petiti Y, Alderete A, Acevedo G. Reconocimiento de la violencia laboral en el sector de la laboral en el sector de la salud. Revista Cubana de Salud y Trabajo. 2012;13(3):7-15.

18. Zafar W, Siddiqui E, Ejaz K, Umer M, Rahim U, Jamali S, Razzak J. Health care personnel an workplace violence in the emergency departments of a volatile metropolis: Results from Karachi, Pakistan. Rev. J. Emerg. Med. 2013; 454(5):76172.

19. Nooran A, Feng J. Workplace violence against nurses in Indonesian emergency departments. Rev. Enferm. Clín. 2018; 28(S1):184-90.

20. Paravic T, Burgos M. Prevalencia de violencia física, abuso verbal y factores asociados en trabajadores/as de servicios de emergencia en establecimientos de salud públicos y privados. Rev Med Chile 2018;146:727-36.

21. Comisión de Conciliación y Arbitraje Médico del Estado de México. Carta de Derechos Generales de las Enfermeras y los Enfermeros. [consultado noviembre de 2018]. Disponible en:http://salud.edomex.gob.mx/ccamem/td_c_der_ generales.html

22. Kalisch B, Landstrom G, Williams R. Missed nursing care: Error of omission. Nurs Outlook. 2009; 57(1):3-9.

23. Harvey C, Thompson S, Pearson M, Willis E, Toffoli L, Missed nursing care as an "art form": The contradictions of nurses as carers. Rev. Nursing inquiry 2017;24(3):1-8. 